\title{
Suppression of CRTC2-mediated hepatic gluconeogenesis by TRAF 6 contributes to hypoglycemia in septic shock
}

\author{
Sihan $\mathrm{Lv}^{1}$, Xinchen Qiu ${ }^{1,2}$, Jian $\mathrm{Li}^{1,2}$, Weida $\mathrm{Li}^{2}$, Chao Zhang ${ }^{2}$, Zhen-Ning Zhang ${ }^{2}$, Bing Luan ${ }^{1}$ \\ ${ }^{1}$ Department of Endocrinology, Shanghai Tenth People's Hospital, School of Medicine, Tongji University, Shanghai, China; \\ ${ }^{2}$ Translational Medical Center for Stem Cell Therapy \& Institute for Regenerative Medicine, Shanghai East Hospital, School of \\ Life Sciences and Technology, Tongji University, Shanghai, China
}

\begin{abstract}
Although hypoglycemia has been documented as a major cause of high mortality in the setting of septic shock, the mechanism of hypoglycemia in infection has not been clearly determined. Hepatic gluconeogenesis serves as an important mechanism to maintain glucose levels under physiological conditions and CREB coactivator CRTC2 plays an important role in regulating gluconeogenic gene expression. Here, we show that triggering of the Toll-like receptor 4 pathway in response to endotoxin lipopolysaccharide (LPS) inhibits gluconeogenic gene expression and hepatic glucose output by

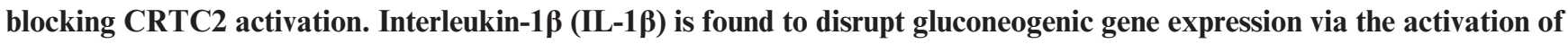
the E3 ubiquitin ligase TRAF6, a key component of the Toll-like receptor 4 signaling pathway that associates with and ubiquitinates CRTC2. TRAF6 promotes the K63-linked ubiquitination of CRTC2, a modification that blocks binding of calcineurin at an adjacent calcineurin-binding site, thereby disrupting CRTC2 dephosphorylation in response to glucagon signals. Mutation of TRAF6-binding sites or ubiquitination site in CRTC2 rescues hepatic gluconeogenesis in LPS-challenged mice. These results suggest that pro-inflammatory signals intersect with the CRTC2 pathway in liver, thus contributing to hypoglycemia caused by infection.
\end{abstract}

Keywords: CRTC; gluconeogenesis; septic shock; TRAF6; ubiquitination

Cell Discovery (2016) 2, 16046; doi:10.1038/celldisc.2016.46; published online 13 December 2016

\section{Introduction}

Hypoglycemia is a cause of considerable morbidity and mortality in the setting of septic shock both in experimental animals and in humans [1-4]. Extensive studies have been conducted to elucidate the mechanisms of hypoglycemia under septic shock, using animal models injected with lipopolysaccharide (LPS). LPS induces hyperglycemia approximately $1 \mathrm{~h}$ after injection [5] followed by hypoglycemia within $6 \mathrm{~h}[6]$ in mice. Glycogen depletion, decreased gluconeogenesis and increased glucose consumption have been suggested as potential mechanisms of hypoglycemia

\footnotetext{
Correspondence: Zhen-Ning Zhang.

Tel: +86 13262825103;

E-mail: znzhang@tongji.edu.cn

or Bing Luan

Tel: +86 13262825126;

E-mail: bluan@tongji.edu.cn

Received 28 September 2016; accepted 10 November 2016
}

induced by LPS [7-9]. However, the detailed signal pathway still remains unsolved.

Under hypoglycemia, increases in circulating glucagon promote hepatic glucose production in part through the activation of gluconeogenesis pathway by CREB coactivator CRTC2 [10]. Sequestered in the cytoplasm via phosphorylation-dependent interactions with 14-3-3 proteins, CRTC2 is dephosphorylated in response to glucagon, when it migrates to the nucleus and binds to CREB over gluconeogenic genes [11]. Glucagon stimulates CRTC2 dephosphorylation via the PKA-dependent inhibition of SIK2 and via the activation of the Ser/Thr phosphatase calcineurin $[12,13]$.

Here we show that in response to endotoxin (LPS) stimulation, inhibition of gluconeogenic gene expression via blocking CRTC2 activation by interleukin-1 $\beta$ (IL-1 $\beta$ ) released from macrophage leads to hypoglycemia in mice. Indeed, TRAF6, the E3 ubiquitin ligase activated by IL-1 $\beta$ associates with and ubiquitinates CRTC2. K63-linked ubiquitination of CRTC2 by 
TRAF6 blocked binding of calcineurin and thereby disrupted CRTC2 dephosphorylation in response to glucagon signals. As mutation of TRAF6-binding sites in CRTC2 rescued hepatic gluconeogenesis in LPS-challenged mice, our results suggest an important regulatory node by which pro-inflammatory signals intersect with the CREB/CRTC2 pathway in liver and perhaps other tissues.

\section{Results}

\section{Pro-inflammatory cytokines inhibit gluconeogenic program}

To monitor effect of LPS on hepatic gluconeogenesis in vivo, we delivered Glucose-6-phosphatase (G6pase)luc or NF-кB-luc adenovirus in mouse liver via tailvein injection and imaged their luciferase reporter activities respectively under fasting conditions. As expected, NF-KB-luc activity increased remarkedly in the liver after LPS injection (Supplementary Figure S1a). By contrast with its stimulatory effects on the NF- $\mathrm{KB}$ pathway, administration of LPS acutely reduced G6pase-luc activity in the liver (Figure 1a). Similarly, the expression of endogenous inflammatory genes including $I L-1 \beta$ and $T N F \alpha$ was induced while the expression of gluconeogenic genes including G6pase and Phosphoenolpyruvate carboxykinase 1 (Pckl) was by contrast inhibited in the liver (Figure $1 \mathrm{~b}$ and Supplementary Figure S1b). As a result, circulating blood glucose concentrations were dramatically decreased (Figure 1c). LPS has been proposed to modulate the gluconeogenic program indirectly via the activation of resident macrophages [14]. Supporting this scenario, exposure of primary hepatocytes to LPS had no effect on G6pase gene expression, whereas medium from LPS-stimulated RAW246.7 macrophages potently reduced it (Supplementary Figure S1c). Based on these results, we hypothesized that LPS-induced cytokine such as IL-1 $\beta$, tumor necrosis factor- $\alpha(\mathrm{TNF} \alpha)$ or IL-6 may inhibit gluconeogensis in hepatocytes. Supporting this notion, exposure of cultured hepatocytes to IL- $1 \beta$, TNF $\alpha$ but not IL-6 downregulated effects of glucagon on gluconeogenic gene expression (G6pase, Pckl) as well as glucose output (Figure 1d-f).

Realizing the importance of the second messenger cAMP in mediating hepatic gluconeogenesis, we tested whether pro-inflammatory cytokines disrupt gluconeogenic gene expression via the CRTC2/CREB pathway. Although exposure to IL-1 $\beta$ or TNF $\alpha$ had no effect on the PKA-mediated phosphorylation of CREB in response to glucagon (Figure $1 \mathrm{~g}$ ), IL-1 $\beta$ or TNF $\alpha$ blocked the dephosphorylation and nuclear shuttle of CRTC2 in hepatocytes exposed to glucagon (Figure 1g and Supplementary Figure S2). Glucagon-stimulated recruitment of CRTC2 to the G6pase promoter was also inhibited by IL-1 $\beta$ or TNF $\alpha$ treatment (Figure $1 \mathrm{~h}$ ). Moreover, adenoviral expression of phosphorylationdefective (S171, 275A) CRTC2 rescued G6pase gene expression and glucose output in primary hepatocytes exposed to IL-1 $\beta$ or TNF $\alpha$, whereas wild-type CRTC2 did not (Figure 1i and $\mathrm{j}$ ).

\section{Pro-inflammatory signals acutely disrupt hepatic gluconeogenesis via TRAF6-mediated inhibition of CRTC2}

TRAF6, a key component of IL-1 $\beta$ signal pathway, has been found to associate with relevant substrates containing a conserved PXEXXAr/Ac (Ar/Ac for an aromatic or acidic residue) motif [15]. We found that CRTC2 contains two well-conserved consensus PXEXXAr/Ac motifs, suggesting its interaction with TRAF6 (Figure 2a). We confirmed the CRTC2: TRAF6 interaction in wild type but not $\mathrm{TRAF}^{-1-}$ or $\mathrm{CRTC2}^{-1-} \mathrm{MEFs}$, under basal conditions and to a greater extent following exposure to IL-1 $\beta$, by co-immunoprecipitation assay of endogenous proteins (Figure 2b). Supporting a functional role for TRAF6, exposure to IL-1 $\beta$ decreased CRTC2/CREB target gene Nuclear receptor subfamily 4, group A, member 2 $(\mathrm{Nr} 4 \mathrm{a} 2)$ expression in wild-type mouse embryonic fibroblasts (MEFs), but it had only modest effects in $\mathrm{TRAF6}^{-1-}$ MEFs or $\mathrm{CRTC2}^{-1-} \mathrm{MEFs}$ co-stimulated with Forskolin (FSK) (Figure 2c). Restoring TRAF6 expression in TRAF6 ${ }^{-1-}$ MEFs or CRTC2 expression in CRTC2 ${ }^{-1-}$ MEFs rescued the effect (Supplementary Figure S3a and b). Furthermore, overexpression of wild-type TRAF6 but not catalytically inactive (C70A) TRAF6 reduced gluconeogenic gene (G6pase, Pckl) expression and glucose output as well as CRTC2 occupancy over the G6pase promoter in hepatocytes exposed to glucagon (Supplementary Figure S4a-d), while RNAi-mediated depletion of TRAF6 increased it (Supplementary Figure $\mathrm{S} 4 \mathrm{e}-\mathrm{h}$ ). Taken together, these results indicate that IL-1 $\beta$ inhibits CRTC2 signaling through TRAF6.

We considered that TRAF6 may promote CRTC2 ubiquitination. Exposure to IL-1 $\beta$ increased CRTC2 ubiquitination in wild type but not $\mathrm{TRAF}^{-1-}$ or $\mathrm{CRTC2}^{-1-}$ MEFs (Figure 2d). Similarly, overexpression of wild type but not TRAF6 C70A potentiated effects of IL- $1 \beta$ on CRTC 2 ubiquitination (Figure 2e), whereas RNAi-mediated depletion of 


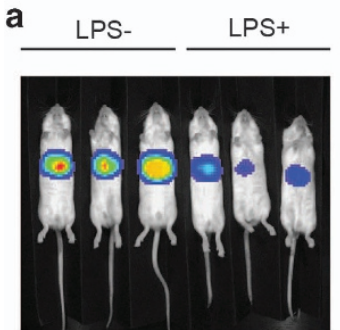

G6pase-luc b

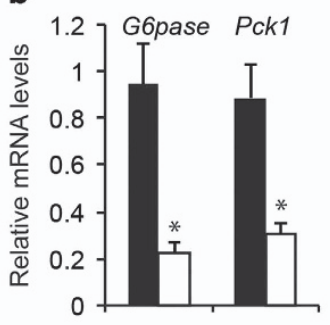

c

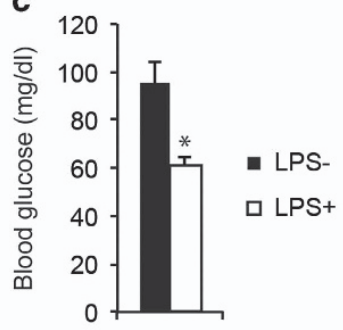

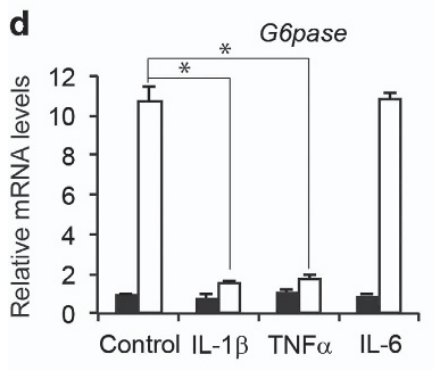

g

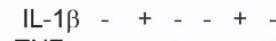

TNF $\alpha$ - $-+{ }_{-}+$

Glucagon - - - + + +

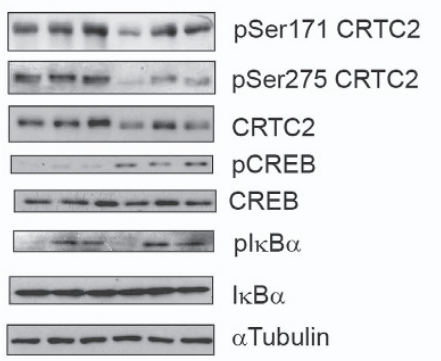

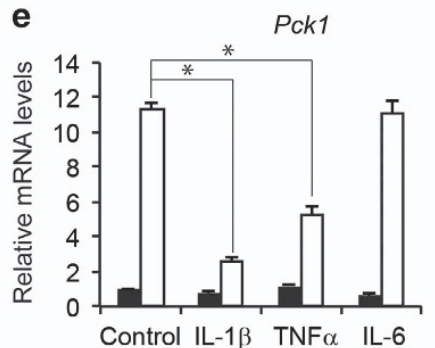

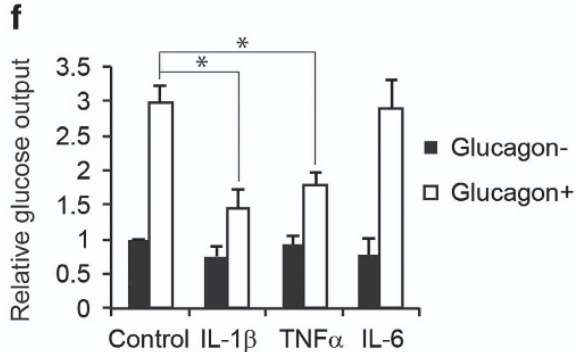

h
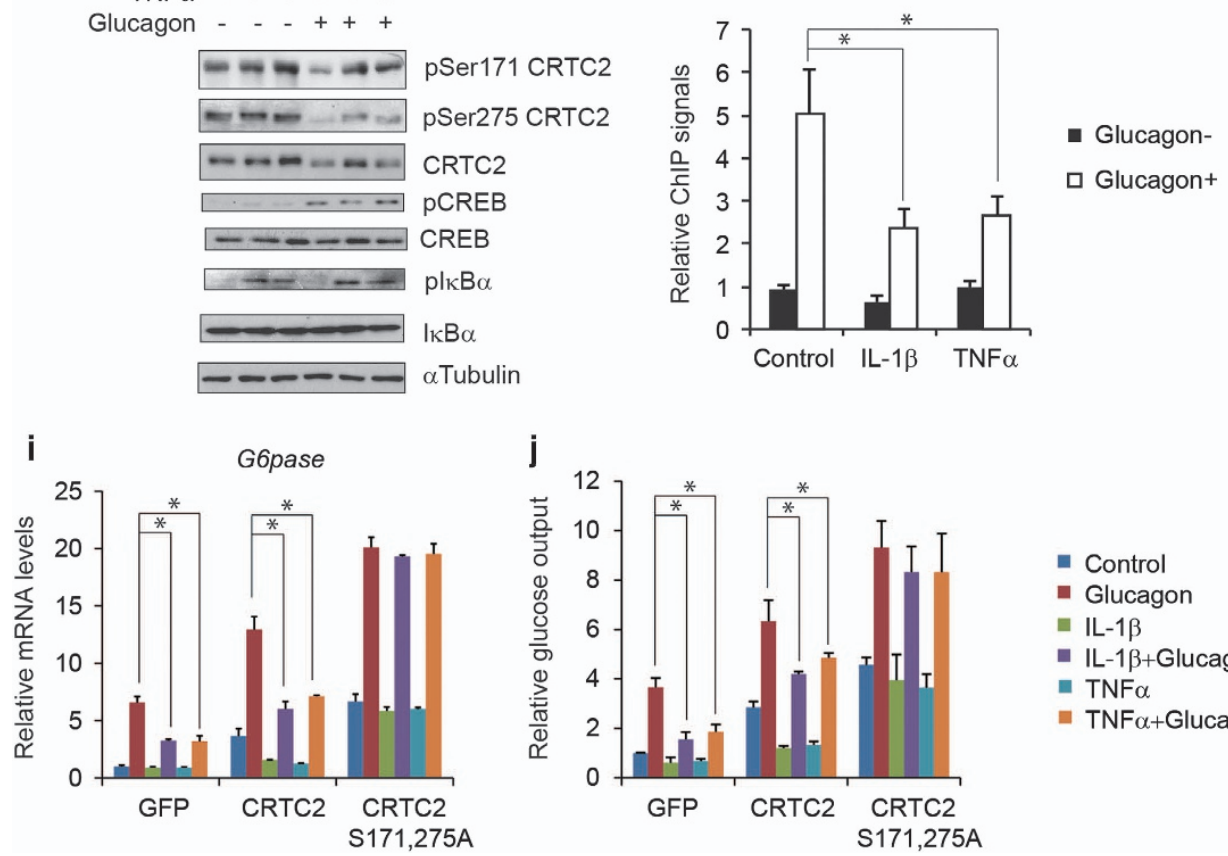

- Control

- Glucagon

- IL-1 $\beta$

- IL-1 $1 \beta+$ Glucagon

- TNF $\alpha$

- TNF $\alpha+$ Glucagon

Figure 1 Pro-inflammatory cytokines inhibit gluconeogenic program. (a-c) Effect of LPS (30 $\left.\mathrm{mg} \mathrm{kg}^{-1}\right)$ administration on hepatic G6pase luciferase reporter activity (a) as well as mRNA amounts for gluconeogenic genes (b), and circulating blood glucose concentrations (c) in fasted mice ( $n=9$ in each group; $\left.{ }^{*} P<0.05\right)$. (d-f) Effect of IL-1 $\beta\left(10 \mu \mathrm{I} \mathrm{I}^{-1}\right)$, TNF $\alpha\left(10 \mu g \mathrm{I}^{-1}\right)$ and IL-6 $\left(10 \mu \mathrm{g} \mathrm{I}^{-1}\right)$ on gluconeogenic gene expression including G6pase (d) and Pck1 (e), as well as glucose output (f) in cultured primary hepatocytes exposed to glucagon $(20 \mathrm{~nm})\left({ }^{\star} P<0.05\right)$. (g) Immunoblot showing effects of IL-1 $\beta\left(10 \mu \mathrm{I} \mathrm{I}^{-1}\right)$ and TNF $\alpha\left(10 \mu \mathrm{g} \mathrm{I} \mathrm{I}^{-1}\right)$ on glucagon (20 nM)-induced CRTC2 dephosphorylation in primary hepatocytes. (h) Effect of IL-1 $\beta\left(10 \mu \mathrm{I} \mathrm{I}^{-1}\right)$ and TNF $\alpha\left(10 \mu \mathrm{g} \mathrm{I}{ }^{-1}\right)$ on CRTC2 occupancy over the G6pase promoter in hepatocytes exposed to glucagon $(20 \mathrm{~nm})\left({ }^{\star} P<0.05\right)$. (i, j) Effect of wild type and phosphorylation-defective S171,275A CRTC2 overexpression on glucagon (20 nm)-induced G6pase mRNA amounts (i) and glucose output $(\mathrm{j})$ in primary hepatocytes exposed to IL-1 $\beta\left(10 \mu \mathrm{g} \mathrm{I}^{-1}\right)$ and TNF $\alpha\left(10 \mu \mathrm{g} \mathrm{I}^{-1}\right)\left({ }^{*} P<0.05\right)$. 
a

\begin{tabular}{|c|c|}
\hline $\begin{array}{c}\text { CREB Binding } \\
\text { Domain }\end{array}$ & $\begin{array}{c}\text { Transactivatio } \\
\text { Domain }\end{array}$ \\
\hline \begin{tabular}{|l|l|}
$\mathrm{CBD}$ & \\
\end{tabular} & \begin{tabular}{l|l|} 
TAD \\
\end{tabular} \\
\hline
\end{tabular}

Homo sapiens CRTC2 (1) 135-LSP PPESSW

Mus musculus CRTC2 (1) 135-LSP PPESSW

Rattus norvegicus CRTC2 (1) 135-LSP PPESGW

Bos taurus CRTC2 (1) 135-LSP PPESSW

Consensus 2

Homo sapiens CRTC2 (2) 291-PLD PEETAY

Mus musculus CRTC2 (2) 291-PLD PEETVY

Rattus norvegicus CRTC2 (2) 290-PLD PEETVY

Bos taurusCRTC2 (2) 290-PLD PEETAY b

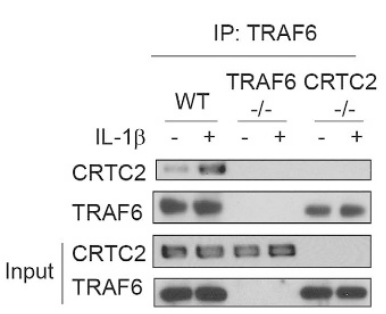

c

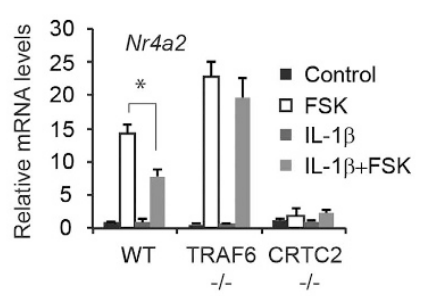

d

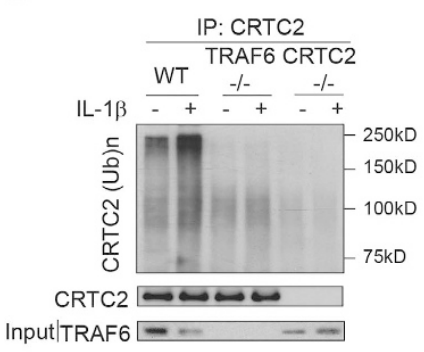

e

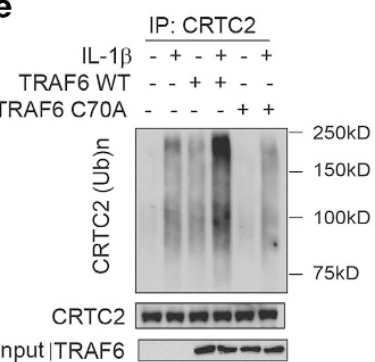

f

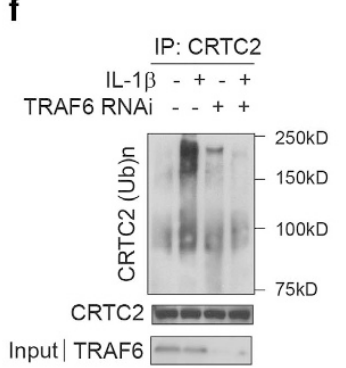

g

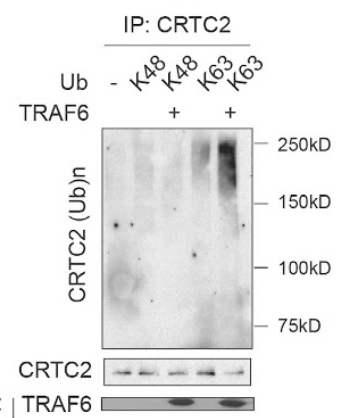

h

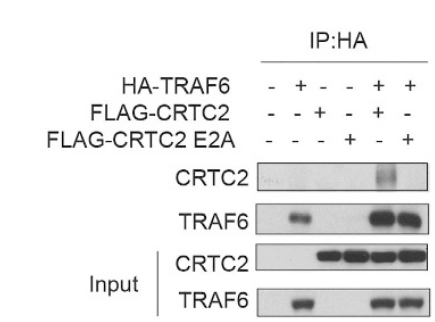

i

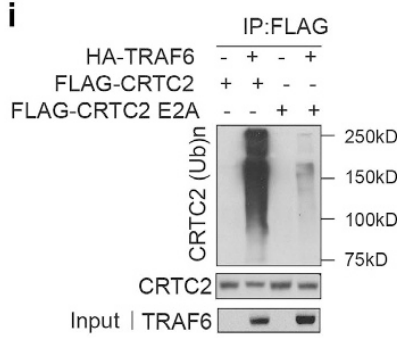

k
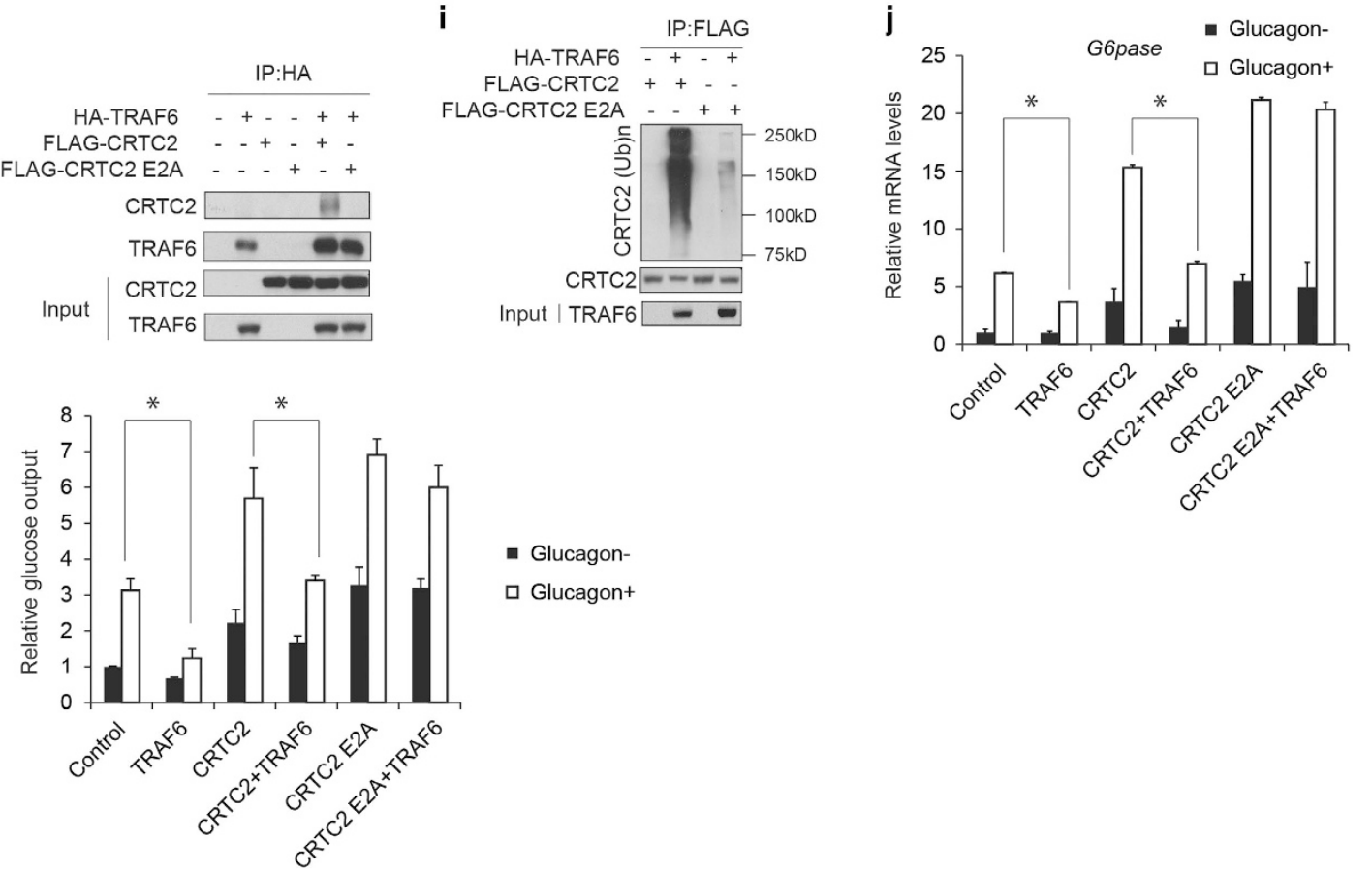

Figure 2 TRAF6 mediates effects of IL-1 $\beta$ on CRTC2 activity. (a) Diagram of CRTC2 and amino acid sequence alignment showing conserved TRAF6-binding motifs (PXEXXAr/Ac) in CRTC2 from different species. (b) Immunoblot of CRTC2 recovered from immunoprecipitates of TRAF6 prepared from wild type, TRAF6 ${ }^{-/-}$or CRTC2 ${ }^{-1-}$ MEFs. Effect of IL-1 $1 \beta\left(10 \mu \mathrm{g} \mathrm{I}^{-1}\right)$ treatment shown. (c) Effect of IL-1 $\beta\left(10 \mu \mathrm{g} \mathrm{I}^{-1}\right)$ on CRTC2 target gene Nr4a2 expression in wild type and TRAF6 ${ }^{-1-}$ or CRTC2 $^{-1-} \mathrm{MEFs}^{\mathrm{CO}-}$ treated with FSK $(10 \mu \mathrm{m})\left({ }^{*} P<0.05\right)$. (d) Immunoblot showing amounts of ubiquitinated CRTC2 recovered from IPs of CRTC2 prepared from wild type and TRAF6 ${ }^{-1-}$ MEFs. (e) Effect of TRAF6 wild type or catalytically inactive (C70A) overexpression on CRTC2 ubiquitination in primary hepatocytes exposed to IL-1 $\beta\left(10 \mu \mathrm{g} \mathrm{I}^{-1}\right)$. (f) Effect of RNAi-mediated depletion of TRAF6 on CRTC2 ubiquitination in primary hepatocytes exposed to IL-1 $\beta\left(10 \mu \mathrm{I} \mathrm{I}^{-1}\right)$. (g) Immunoblot showing effect of TRAF6 on amounts of ubiquitinated CRTC2 in HEK293T cells co-expressing mutant ubiquitins with mutation of all lysines except K48 or K63. (h) Immunoblots showing effect of mutations in the TRAF6-binding motifs (E2A) in CRTC2 on its association with TRAF6 in HEK293T cells. (i) Immunoblots showing effect of mutations in the TRAF6-binding motifs (E2A) in CRTC2 on its ubiquitination in HEK293T cells. (j, k) Effect of TRAF6 overexpression on wild type and E2A CRTC2 induced G6pase mRNA amounts (j) and glucose output $(\mathbf{k})$ in primary hepatocytes exposed to glucagon $(20 \mathrm{~nm})\left({ }^{*} P<0.05\right)$. 
TRAF6 reduced amounts of ubiquitinated CRTC2 (Figure 2f) in primary hepatocytes.

E3 ubiquitin ligases have been shown to regulate substrate degradation or cellular trafficking via K48 or K63-linked ubiquitination, respectively [16-18]. Consistent with its effects on other cellular proteins, TRAF6 appeared to ubiquitinate CRTC2 selectively via K63-dependent linkages; overexpression of K63-only ubiquitin promoted TRAF6-dependent ubiquitination of CRTC2 while K48-only mutant ubiquitin did not (Figure 2g).

Within the CRTC family, CRTC1 and CRTC2 contain well-conserved consensus PXEXXAr/Ac motifs, whereas CRTC3 does not (Supplementary Figure S5a). Correspondingly, TRAF6 was found to associate with and promote the ubiquitination of both CRTC1 and CRTC2 but not CRTC3 (Supplementary Figure S5b). As a result, overexpression of TRAF6 reduced G6pase reporter activity induced by both CRTC1 and CRTC2 but not CRTC3 (Supplementary Figure S5c). Moreover, mutation of the putative interaction motifs in CRTC1 and CRTC2 disrupted TRAF6 binding and ubiquitination (Figure $2 \mathrm{~h}$ and $\mathrm{i}$ and Supplementary Figure S5d and e). Consistently, TRAF6 reduced G6pase gene expression or reporter activity induced by wild-type CRTC 1 and CRTC 2 but not TRAF6-interaction-defective CRTC1 and CRTC2 (Figure $2 \mathrm{j}$ and Supplementary Figure S5f). As a result, glucose output induced by wild-type CRTC2 but not TRAF6-interaction-defective CRTC2 was inhibited by TRAF6 (Figure 2k).

TRAF2, a counterpart of TRAF6 in TNF $\alpha$ signal pathway, also interacted with CRTC2 and promoted ubiquitination of CRTC2 (Supplementary Figure S6a and b). Similarly, overexpression of wild-type TRAF2 but not catalytically inactive (C34A) TRAF2 reduced G6pase reporter activity (Supplementary Figure S6c).

Based on these results, we evaluated whether TRAF6 mediates inhibitory effects of LPS on the gluconeogenic program in vivo. LPS administration (i.p.) decreased G6pase-luc activity as well as gluconeogenic gene expression (G6pase and Pckl) and circulating blood glucose concentrations in fasted mice; these effects were potentiated by overexpression of wild type but not catalytically inactive C70A mutant TRAF6 (Figure 3a-d). Conversely, RNAi-mediated knockdown of TRAF6 enhanced G6pase-luc activity as well as gluconeogenic gene expression (G6pase and $P(k 1)$ and circulating glucose concentrations in fasted mice, indicating that TRAF6 modulates hepatic gluconeogenesis in part through inhibition of CRTC2 (Figure $3 \mathrm{e}-\mathrm{h}$ ). Pointing to an important role for the
TRAF6:CRTC2 interaction, effects of LPS on the gluconeogenic profile were diminished in mice expressing TRAF6-interaction-defective CRTC2 relative to wild-type CRTC2 (Figure 3i-1). Collectively, these results suggest that TRAF6 mediates inhibitory effects of a pro-inflammatory signal on CRTC2 activity in liver.

\section{TRAF6-mediated ubiquitination of CRTC2 disrupts its dephosphorylation via $\mathrm{CnA}$}

We examined the mechanism by which TRAF6mediated ubiquitination inhibits CRTC2 activity. Based on mass spectrometry studies showing that CRTC2 undergoes ubiquitination at Lys628 [11], we tested whether this site is ubiquitinated by TRAF6. Supporting this idea, exposure to IL-1 $\beta$ increased CRTC2 ubiquitination in hepatocytes expressing wildtype CRTC2, but it had no effect in cells expressing K628R mutant CRTC2 (Figure 4a). Correspondingly, exposure to IL-1 $\beta$ reduced glucagon-stimulated G6pase gene expression in cells expressing wild-type CRTC2, but not in K628R CRTC2-expressing cells (Figure 4b). Furthermore, while exposure to IL-1 $\beta$ had only modest effects on CRTC2/CREB target gene $\mathrm{Nr} 4 \mathrm{a} 2$ expression in $\mathrm{CRTC}^{-/-}$MEFs co-stimulated with FSK, overexpression of wild-type CRTC2 but not CRTC2 E2A or CRTC2 KR in CRTC2 ${ }^{-1-}$ MEFs rescued the effect (Supplementary Figure S3a). As a result, K628R mutant CRTC2 rescued hepatic G6pase reporter activity as well as gluconeogenic gene expression (G6pase and Pckl) and circulating blood glucose concentrations in mice administered LPS, whereas wildtype CRTC2 did not (Figure $4 \mathrm{c}-\mathrm{g}$ ). It has been reported that constitutive photomorphogenic protein 1 (COP1) interacts with CRTC2 and promotes the K48 ubiquitination and degradation of CRTC2 at Lys628 [12]. However, COP1 interaction-defective CRTC2 mutant (CRTC2 V214, P215A, CRTC2 VP/AA) was still able to be ubiquitinated by TRAF6 and exposure to IL-1 $\beta$ reduced glucagon-stimulated G6pase gene expression in cells expressing either wild-type CRTC2 or VP/AA CRTC2 (Supplementary Figure $\mathrm{S} 7 \mathrm{a}$ and $\mathrm{b}$ ). Furthermore, IL-1 $\beta$ induced CRTC2 ubiquitination at a similar level in either USi-infected or COP1i-infected cells (Supplementary Figure S7c). Consistently, when primary hepatocytes were infected with adenovirus encoding either unspecific RNAi (USi) or COP1 RNAi (COP1i), exposure to IL-1 $\beta$ reduced glucagon-stimulated G6pase gene expression in cells expressing either USi or COP1i (Supplementary Figure S7d), excluding COP1 participation in this setting. Taken together, these results 


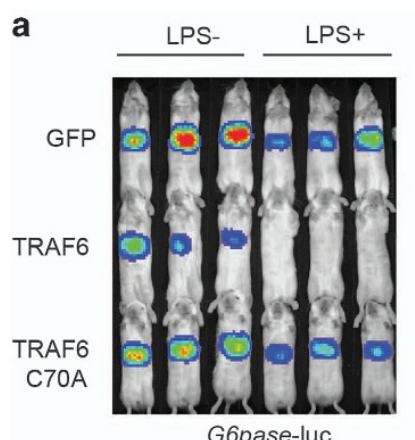

b

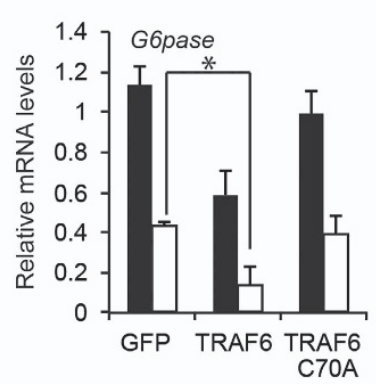

C

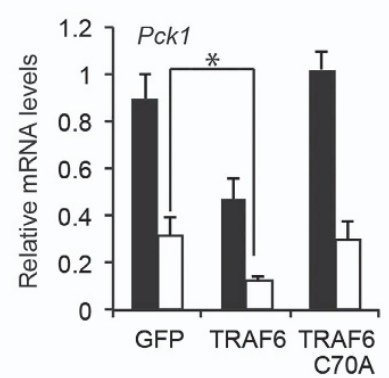

d

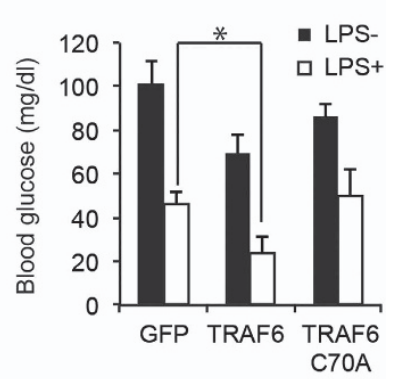

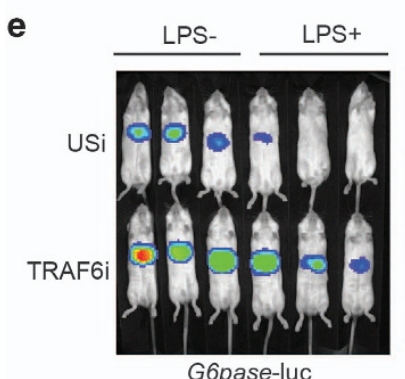

G6pase-luc

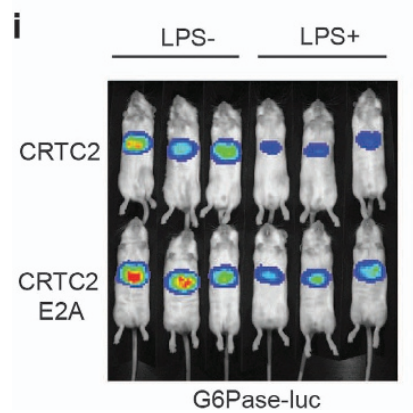

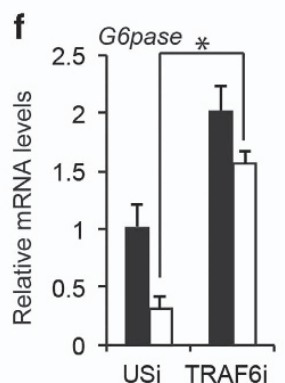

j

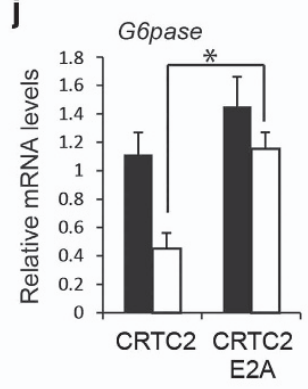

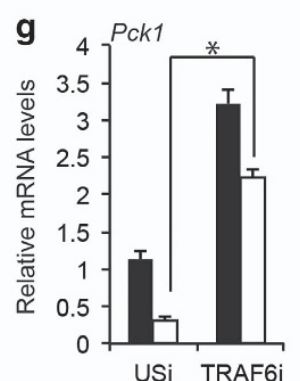

k

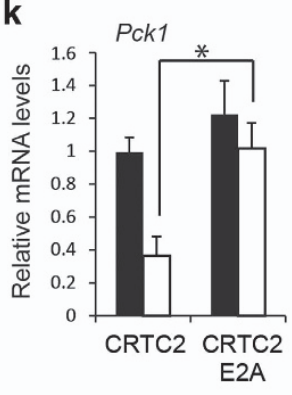

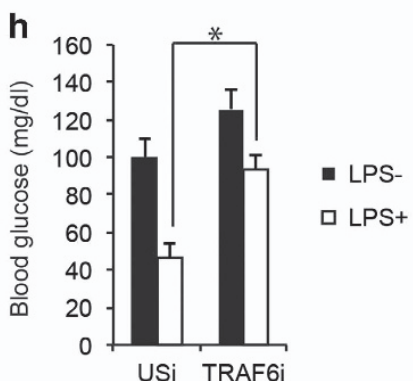

I

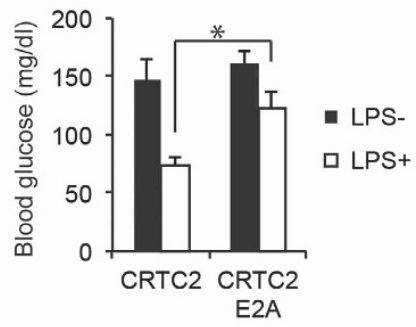

Figure 3 Pro-inflammatory signals acutely disrupt hepatic gluconeogenesis via TRAF6-mediated inhibition of CRTC2. (a-d) Effect of adenoviral TRAF6 wild type or catalytically inactive C70A overexpression on hepatic G6pase reporter activity (a), as well as mRNA amounts for gluconeogenic genes including G6pase (b) and Pck1 (c) and circulating blood glucose concentrations (d) in fasted mice following administration of LPS $\left(30 \mathrm{mg} \mathrm{kg}^{-1}\right)\left(n=9\right.$ in each group; $\left.{ }^{*} P<0.05\right)$. (e-h) Effect of adenoviral RNAi-mediated knockdown of TRAF6 on hepatic G6pase reporter activity (e), as well as mRNA amounts for gluconeogenic genes including G6pase (f) and Pck1 (g), and circulating blood glucose concentrations (h) in fasted mice following administration of LPS $\left(30 \mathrm{mg} \mathrm{kg}^{-1}\right)\left(n=9\right.$ in each group; $\left.{ }^{*} P<0.05\right)$. (i-I) Effect of wild type and TRAF6 interaction-defective CRTC2 (E2A) on hepatic G6pase reporter activity (i) as well as mRNA amounts for gluconeogenic genes including G6pase (j) and Pck1 (k), and circulating blood glucose concentrations (I) in fasted mice following administration of LPS (30 mg kg $\left.{ }^{-1}\right)$ $\left(n=9\right.$ in each group; $\left.{ }^{*} P<0.05\right)$.

indicate that pro-inflammatory cytokines inhibit the CRTC2 pathway via TRAF6-dependent ubiquitination of CRTC2 at Lys628.

Glucagon has been shown to promote CRTC2 dephosphorylation via induction of calcineurin ( $\mathrm{CnA})$ [19]. Interestingly, Lys628 is located adjacent to a calcineurin-binding site (613-PNIILT-618) in CRTC2 (Figure 5a), leading us to consider that TRAF6dependent ubiquitination might interfere with $\mathrm{CnA}$ binding and dephosphorylation of CRTC2. In co-immunoprecipitation studies, exposure to IL-1 $\beta$ decreased the association of $\mathrm{CRTC} 2$ with $\mathrm{CnA}$ and correspondingly blocked the dephosphorylation of CRTC2 at the Ser171 and Ser275 sites (Figure 5b and c). In contrast with wild-type CRTC2, IL-1 $\beta$ had no effect on $\mathrm{CnA}$ binding or dephosphorylation of ubiquitination-defective K628R CRTC2 (Figure 5b and c). Similarly, substitution of the native CnAbinding site (PNIILT) in CRTC2 with an optimized high affinity site (PVIVIT) [20, 21] increased CnA binding and CRTC2 dephosphorylation even in the presence of IL-1 $\beta$ co-stimulation (Figure $5 \mathrm{~d}$ and e). 
a

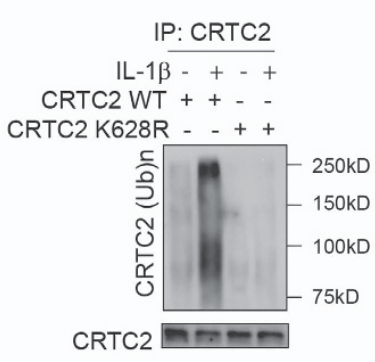

d

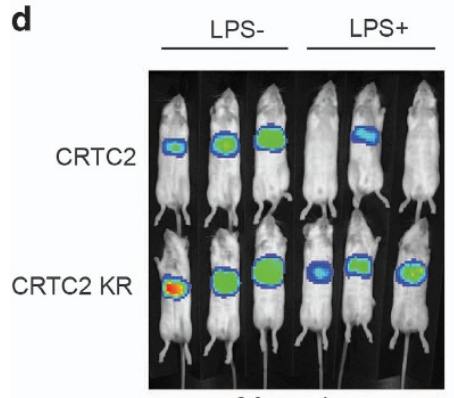

G6pase-luc
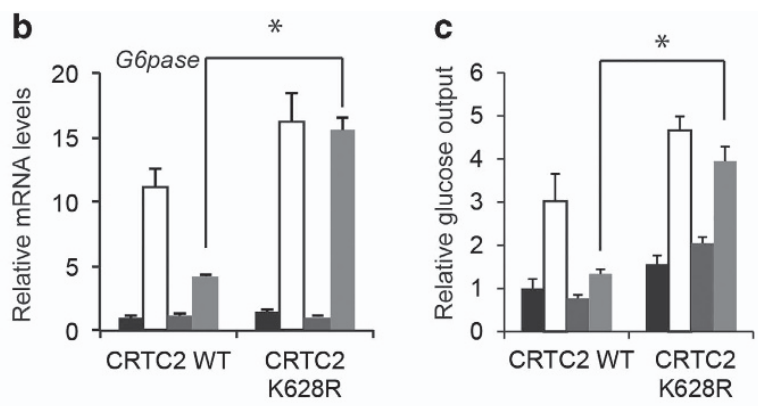

- Control

$\square$ Glucagon

- IL-1 $1 \beta$

- IL-1 $1 \beta+$ Glucagon
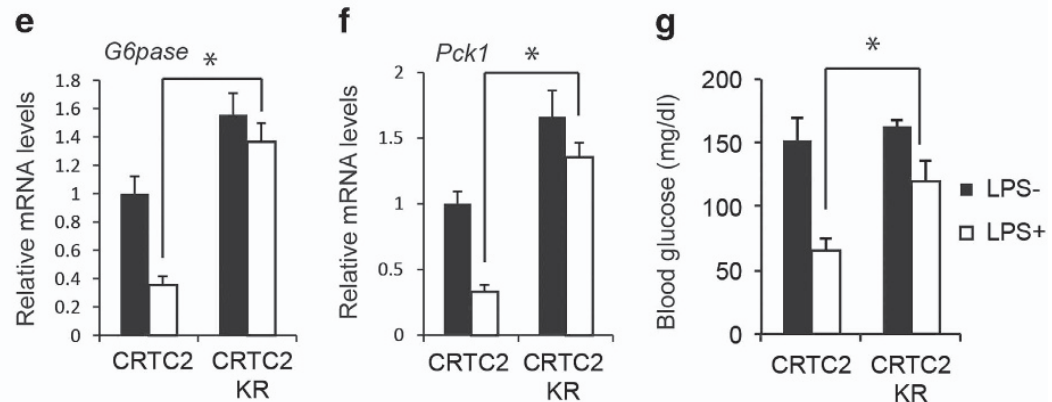

Figure 4 TRAF6-mediated ubiquitination of CRTC2 inhibits its activity. (a) Immunoblot showing ubiquitination of wild type and K628R mutant CRTC2 in hepatocytes exposed to IL-1 $\beta\left(10 \mu \mathrm{I} \mathrm{I}^{-1}\right)$. (b, c) Effects of wild type or ubiquitination-defective K628R CRTC2 on G6pase mRNA amounts (b) and glucose output (c) in hepatocytes exposed to IL-1 $\beta\left(10 \mu \mathrm{g} \mathrm{I}^{-1}\right)$ and glucagon (20 nM) $\left({ }^{*} P<0.05\right)$. ( $\left.\mathbf{d}-\mathbf{g}\right)$ Effect of wild type or K628R mutant CRTC2 on hepatic G6pase reporter activity (d) as well as mRNA amounts for gluconeogenic genes including G6pase (e) and Pck1 (f), and circulating blood glucose concentrations (g) in fasted mice following administration of LPS $\left(30 \mathrm{mg} \mathrm{k}^{-1} \mathrm{~g}\right)\left(n=9\right.$ in each group; $\left.{ }^{*} P<0.05\right)$.

As a result, exposure to IL-1 $\beta$ reduced G6pase gene expression as well as glucose output induced by wild-type CRTC2 but not PVIVIT mutant CRTC2 in primary hepatocytes (Figure 5f and g). Collectively, these results demonstrate that pro-inflammatory stimuli disrupt signaling through the CRTC2 pathway in part via the TRAF6-mediated ubiquitination and inhibition of CRTC2 dephosphorylation.

\section{Discussion}

During hypoglycemia, increases in circulating glucagon trigger CRTC2 dephosphorylation in part by increasing its association with $\mathrm{CnA}$ at a consensus PXIXIT motif [19]. Here we show that in response to pro-inflammatory cytokines, TRAF6 stimulates CRTC2 ubiquitination at Lys628, which in turn blocks CRTC2 activation by disrupting CnA binding (Figure 5h). Most importantly, RNAimediated knockdown of TRAF6 or expression of TRAF6-interaction-defective CRTC2 and TRAF6ubiquination-defective CRTC2 in liver rescues the hypoglycemic phenotype induced by LPS injection. Taken together, our results unveil a novel mechanism by which pro-inflammatory signals intersect with the CRTC2 pathway in liver and this mechanism contributes to the hypoglycemia caused by infection.

Post-transcriptional modification at Lys628 of CRTC2 plays important roles in regulating CRTC2 functions. COP1 mediates CRTC2 K48 ubiquitination at Lys628 and promotes CRTCs degradation under feeding signal such as insulin stimulation, which serves as a mechanism for insulin-mediated suppression of gluconeogenesis [12]. On the contrary, fasting signal such as glucagon stimulation activates p300/CBP and p300/CBP in turn acetylates CRTC2 at Lys628, which protects CRTC2 against ubiquitin-mediated degradation [11]. Collectively, these mechanisms help to maintain normal glucose levels via counterregulatory effects of insulin and glucagon on hepatic gluconeogenesis and dysfunction of these mechanisms leads to hyperglycemia in diabetes. Here, our work contributes to the importance of Lys628 modification on CRTC2 functions by showing that inflammation signal stimulates K63 ubiquitination of Lys628 through TRAF6. Although K63 ubiquitination of CRTC2 does not lead to protein degradation, it blocks the binding of CnA to CRTC2 and dephosphorylation 
a

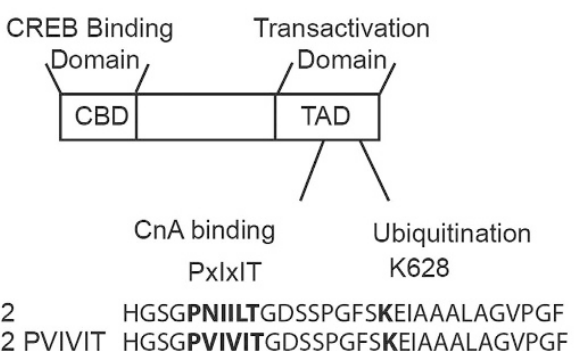

CRTC2 PVIVIT HGSGPVIVITGDSSPGFSKEIAAALAGVPGF

c

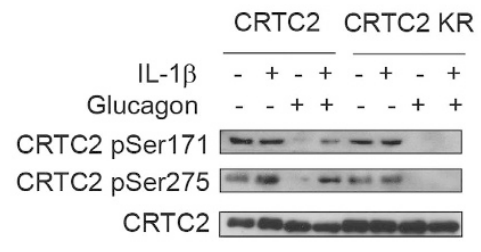

e

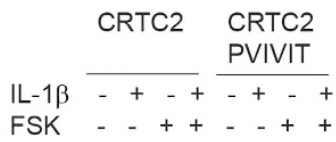

CRTC2 pSer171

CRTC2 pSer275

CRTC2
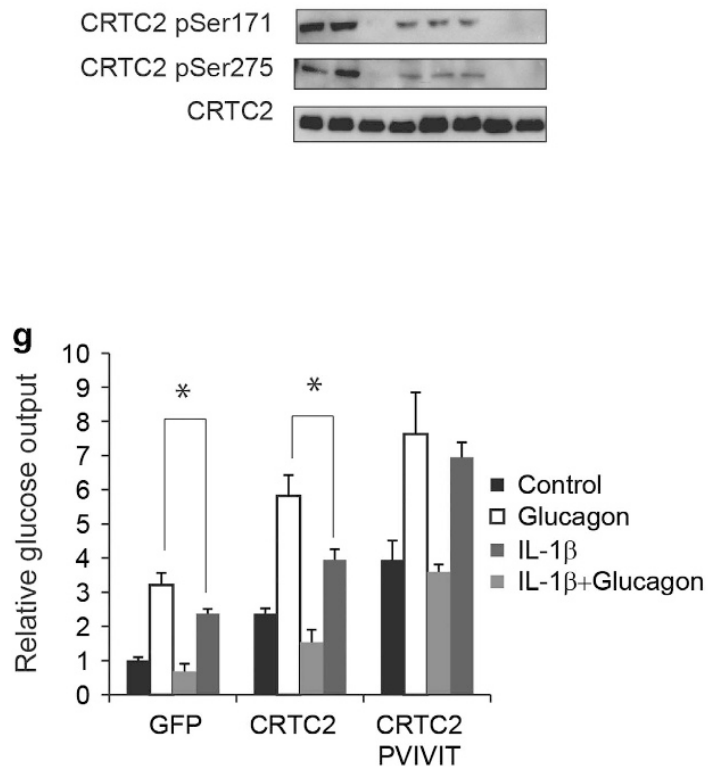

b

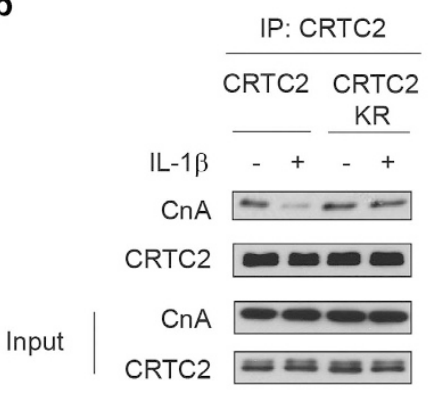

d IP: CRTC2

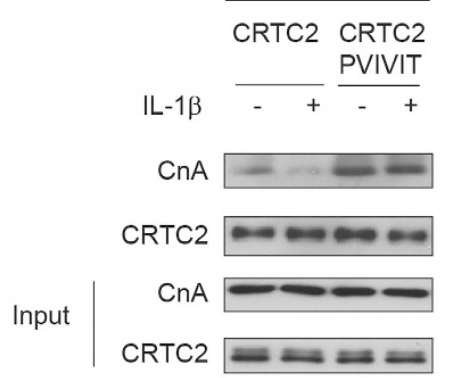

f

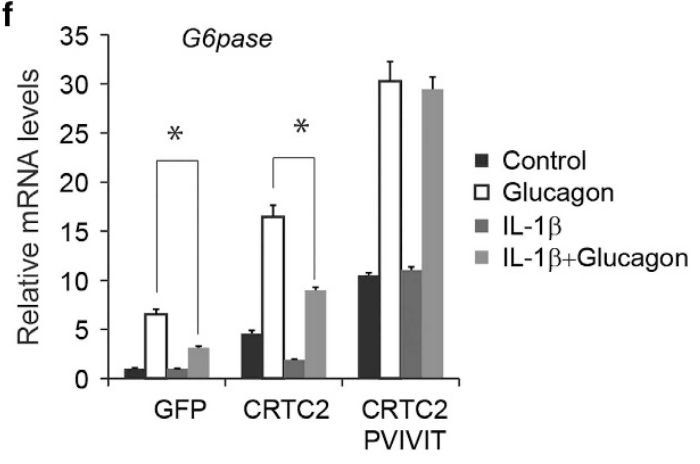

h

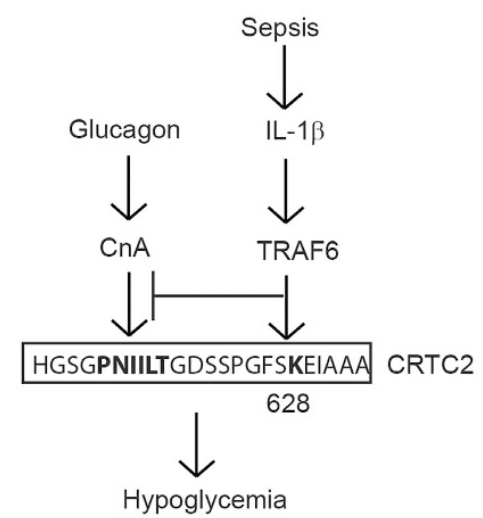

Figure 5 TRAF6-mediated ubiquitination of CRTC2 disrupts its dephosphorylation via CnA. (a) Diagram and sequence of CRTC2 CnA-binding sites and ubiquitination site. (b) Effect of IL-1 $1 \beta\left(10 \mu \mathrm{I} \mathrm{I}^{-1}\right)$ on CnA binding of wild type or K628R mutant CRTC2. (c) Effect of IL-1 $\beta\left(10 \mu \mathrm{g} \mathrm{I}^{-1}\right)$ on dephosphorylation of wild type or K628R mutant CRTC2 in primary hepatocytes exposed to glucagon (20 nM). (d) Effect of IL-1 $\left(10 \mathrm{\mu g} \mathrm{I}^{-1}\right)$ on CnA binding of wild-type CRTC2 or mutant CRTC2 containing a high affinity CnA-binding site (PVIVIT). (e) Effect of IL-1 $\left(10 \mu \mathrm{gl}^{-1}\right)$ on dephosphorylation of wild type or PVIVIT CRTC2 in primary hepatocytes exposed to glucagon (20 nM). (f, g) Effect of wild type and PVIVIT CRTC2 on G6pase mRNA amounts (f) and glucose output $(\mathbf{g})$ in hepatocytes exposed to IL-1 $\beta\left(10 \mu \mathrm{g} \mathrm{I}{ }^{-1}\right)$ and glucagon $(20 \mathrm{nM})\left({ }^{*} P<0.05\right)$. (h) Schematic of proposed mechanism. In response to septic shock, pro-inflammatory cytokines such as IL-1 $\beta$ stimulates CRTC2 ubiquitination at Lys628 through TRAF6, which in turn blocks CRTC2 activation by disrupting CnA binding. 
of CRTC2. Further investigation on Lys628 methylation or sumoylation will unveal more physiological stimulus that could regulate gluconeogensis through CRTC2.

It has been reported that endotoxin-induced hypoglycemia is paralleled by an increase in counteregulatory hormones such as glucagon as well as gluconeogenic precursor supply (lactate and gluconeogenic amino acids) [22, 23]. However, hepatic gluconeogenesis is severely impaired under the same settings, representing a 'glucagon resistance' phenotype [24-26]. In the present study, we show that the glucagon-stimulated signal pathway is defective due to the impaired dephosphorylation of CRTC2 by TRAF6 ubiquitination, a situation quite similar to the impaired insulin signal transduction under insulin resistance status. Thus, acute septic shock causes hypoglycemia via induction of glucagon resistance, while chronic low-grade inflammation leads to hyperglycemia via induction of insulin resistance. The detailed mechanism differences between these two pathological conditions still remain unclear.

Glucocorticoid receptor signaling plays an important role in the regulation of gluconeogenesis [27] and is inhibited by septic shock, although the mechanism has not been clarified $[6,28]$. CRTC2 functions as a coactivator for the glucocorticoid receptor and is required for the glucocorticoid-associated induction of hepatic gluconeogenesis [29]. Besides, glucose uptake in periphery tissue including skeletal muscle and adipose tissue helps to maintain euglycemia under physiological conditions. Glucose uptake is increased following LPS challenge, which contributes to the hypoglycemia caused by infection [30-32]. CRTC2 is reported to inhibit glucose transporter type 4 (Glut4) expression and glucose uptake through ATF3 [33]. Thus, TRAF6-mediated inhibition of CRTC2 may serve as a broad mechanism of septic shock-induced hypoglycemia in multiple tissues, which will need further investigation.

\section{Materials and Methods}

\section{Cells, antibodies and reagents}

Primary hepatocytes were prepared as described [34]. HEK293T and RAW 264.7 (mouse leukemic monocyte macrophage cell line) cells were obtained from the American Type Culture Collection (ATCC, Manassas, VA, USA) and maintained in Dulbecco's modified Eagle's medium with 10\% fetal bovine serum. RAW 264.7 cells were stimulated with LPS for $16 \mathrm{~h}$. Fetal bovine serum-free medium supernatants were collected and supplied to mouse primary hepatocytes. MEFs from wild type and $\mathrm{CRTC} 2^{-l-}$ mice were prepared as previously described [35]. MEFs from wild type and TRAF6 ${ }^{-1-}$ mice were kindly provided by Dr Hui-Kuan Lin (The University of Texas MD Anderson Cancer Center, Houston, TX, USA). All cells were recently authenticated and tested for contamination. Anti-pSer171 CRTC2 (1:1 000), pSer275 CRTC2 (1:1 000), CRTC2 (1:5 000) antibodies were kindly provided by Dr Marc Montminy (Salk Institute, La jolla, CA, USA) [12, 36]. Anti-pSer 32/36 ІкB $\alpha$ (1:1 000; cat. no. 2859), ІкB $\alpha$ (1:1 000; cat. no. 4812), TRAF6 (1:1 000; cat. no. 8028), Ubiquitin (1:1 000; cat. no. 3936) and CnA (1:1 000; cat. no. 2614) antibodies were purchased from Cell signaling Technology (Danvers, MA, USA). Anti- $\alpha$-tubulin (1:5 000; cat. no. ab18251) antibody was purchased from Abcam (Cambridge, UK).

\section{Animals and adenovirus}

Eight- to 10-week-old and weight-matched male C57BL/6J mice were purchased from Shanghai Laboratory Animal Center (Shanghai, China) and were adapted to colony cages with $12 \mathrm{~h}$ light/dark cycle in a temperature-controlled environment with free access to water and standard irradiated rodent diet (5\% fat; Research Diet, D12450, New Brunswick, NJ, USA). For adenovirus injection, $10^{9}$ plaque-forming units (p.f.u.) Ad-G6pase-luc or Ad-NF-кB-luc and $5 \times 10^{7}$ p.f.u. Ad-RSV- $\beta$ gal, $1 \times 10^{8}$ p.f.u. Ad-GFP, Ad-TRAF6, Ad-unspecific RNAi (USi), Ad-TRAF6 RNAi (TRAF6i), Ad-CRTC2, Ad-CRTC2 E2A and Ad-CRTC2 K628R were delivered by tail-vein injection. TRAF6 RNAi was constructed using the sequence 5'-GGGCGAGCTCAAACGGACCATT-3'. All animal studies were approved by the Animal Experiment Committee of Tongji University and in accordance with the guidelines of School of medicine, Tongji University.

\section{In vivo imaging}

Mice were imaged on days 3-5 after adenovirus delivery. Mice were fasted for $12 \mathrm{~h}$ starting at Zeitgeber time (ZT) 12 and injected intraperitoneally with LPS $\left(30 \mathrm{mg} \mathrm{kg}^{-1}\right)$ for $1 \mathrm{~h}$. Before imaging, mice were injected intraperitoneally with $50 \mathrm{mg} \mathrm{kg}^{-1}$ Nembutal (Abbott Laboratories, Lake Bluff, IL, USA) and $100 \mathrm{mg} \mathrm{kg}^{-1}$ sterile firefly D-luciferin (Qianchen, Shanghai, China). Mice were imaged using the IVIS 100 Imaging System (PerkinElmer, Waltham, MA, USA) and image data were analyzed using Living Image software (Xenogen, Hudson, NY, USA) as described [11]. Liver samples were taken immediately after imaging and lysates were subjected to $\beta$-galactosidase assay to ensure the equal level of adenoviral infection.

\section{In vitro analysis}

Mouse tissues were sonicated at $4{ }^{\circ} \mathrm{C}$, centrifuged and supernatants were reserved for $\beta$-gal activity, protein determinations, SDS-PAGE analysis and quantitative analysis. Blood glucose levels were measured with a One Touch Ultra Glucometer (Johnson \& Johnson, New Brunswick, NJ, USA).

\section{Glucose output}

Glucose output from primary hepatocytes was determined enzymatically. Cells were pre-treated with IL-1 $\beta\left(10 \mu \mathrm{g}^{-1}\right)$, TNF $\alpha\left(10 \mu \mathrm{g}^{-1}\right)$ and IL-6 $\left(10 \mu \mathrm{g}^{-1}\right)$ for $15 \mathrm{~min}$ and then stimulated by glucagon $(20 \mathrm{~nm})$ or FSK $(10 \mu \mathrm{M})$ for $1 \mathrm{~h}$ in 
glucose-free M199 media supplemented with $10 \mathrm{~mm}$ lactate and $1 \mathrm{~mm}$ pyruvate. Glucose levels in the medium was measure by Glucose (GO) Assay Kit from Sigma-Aldrich (St Louis, MO, USA)

\section{Quantitative real-time-PCR and immunoblot}

Cells were pre-treated with IL-1 $\beta \quad\left(10 \mu \mathrm{g}^{-1}\right), \quad \mathrm{TNF} \alpha$ $\left(10 \mu \mathrm{g}^{-1}\right)$ and IL-6 $\left(10 \mu \mathrm{g}^{-1}\right)$ for $15 \mathrm{~min}$ and then stimulated by glucagon $(20 \mathrm{~nm})$ or FSK $(10 \mu \mathrm{M})$ for $2 \mathrm{~h}$. Total RNA was isolated by using TRIzol reagent (Invitrogen, Carlsbad, CA, USA) and reverse transcription was done using FastQuant RT kit (Tiangen, Shanghai, China). Real-time PCR was carried out using SuperReal SYBR Green kit (Tiangen Shanghai, China) and Lightcycler 96 (Roche, Penzberg, Germany). $\beta$-Actin was used as a reference gene. Cells were pre-treated with IL-1 $\beta$ $\left(10 \mu \mathrm{g}^{-1}\right), \mathrm{TNF} \alpha\left(10 \mu \mathrm{g} \mathrm{l^{-1 }}\right)$ and IL-6 $\left(10 \mu \mathrm{g}^{-1}\right)$ for $15 \mathrm{~min}$ and then stimulated by glucagon $(20 \mathrm{~nm})$ or FSK $(10 \mu \mathrm{M})$ for $30 \mathrm{~min}$. Immunoblot and immunoprecipitation were performed as described [37].

\section{CRTC2 ubiquitination}

In vivo ubiquitination assays were performed as described [12]. In brief, cells treated with IL-1 $\beta\left(10 \mu \mathrm{g}^{-1}\right)$ for $30 \mathrm{~min}$ were lysed by lysis buffer containing $100 \mathrm{~mm}$ Tris-HCl, $\mathrm{pH}$ 8.0, and $6 \mathrm{M}$ urea. Lysates were diluted and anti-CRTC2 immunoprecipitates were prepared. Ubiquitinated CRTC2 was detected by immunoblot.

\section{Chromatin immunoprecipitation}

Cultured mouse primary hepatocytes were plated in $150 \mathrm{~mm}$ plates and pre-treated with IL-1 $\beta\left(10 \mu \mathrm{g}^{-1}\right)$ or TNF $\alpha\left(10 \mu \mathrm{g} \mathrm{l}^{-1}\right)$ for $30 \mathrm{~min}$, then exposed to glucagon for $1 \mathrm{~h}$. Chromatin immunoprecipitation assays were performed as described previously, with minimal modification [37]. In brief, the treated hepatocytes were crosslinked with $1 \%$ formaldehyde for $15 \mathrm{~min}$. Crosslinking reactions were stopped with $0.125 \mathrm{M}$ glycine. Crosslinked cells were washed in phosphate-buffered saline three times and stored at $-80{ }^{\circ} \mathrm{C}$ before use. Fragmented, precleared chromatin lysate was incubated overnight with indicated antibodies. The primers for G6pase promoter (5'-GGAGGGCAGC CTCTAGCACTGTCAA-3'， 5'-TCAGTCTGTAGGTCAAT CCAGCCCT-3') were used for chromatin immunoprecipitation analysis as described [38]. All signals were normalized to the input chromatin signals.

\section{Statistical analysis}

All studies were performed on at least three independent occasions. Results are reported as mean \pm s.e.m. For all viability experiments, a two-tailed Student's $t$-test or two-way Anova test was used to evaluate statistical significance, which was noted when $* P<0.05$ or $* * P<0.01$.

\section{Conflict of Interest}

The authors declare no conflict of interest.

\section{Acknowledgements}

We thank Dr Marc Montminy and Susan Hedrick at Salk Institute, La jolla, CA, USA, for discussion and technical support. We thank Dr Jerome V Karpiak (University of California San Diego School of Medicine, Sanford Consortium for Regenerative Medicine, La Jolla, CA, USA) for English-language editing. This research was supported by grants from 1,000 Talents Program for Young Scholars of China to BL.

\section{Author contributions}

SL, JL, XQ, ZNZ and BL designed and conducted the experiment and analyzed the data. BL wrote the manuscript. WL, CZ and ZNZ designed the experiments and edited the manuscript. All authors read and approved the manuscript. $\mathrm{BL}$ is responsible for the integrity of the work as a whole.

\section{References}

1 Berk JL, Hagen JF, Beyer WH, Gerber MJ. Hypoglycemia of shock. Ann Surg 1970; 171: 400-408.

2 Ssekitoleko R, Jacob ST, Banura P et al. Hypoglycemia at admission is associated with inhospital mortality in Ugandan patients with severe sepsis. Crit Care Med 2011; 39: 2271-2276.

3 Fischer KF, Lees JA, Newman JH. Hypoglycemia in hospitalized patients. Causes and outcomes. $N$ Engl J Med 1986; 315: 1245-1250.

4 Miller SI, Wallace RJ Jr, Musher DM, Septimus EJ, Kohl S, Baughn RE. Hypoglycemia as a manifestation of sepsis. Am J Med 1980; 68: 649-654.

5 Sugita H, Kaneki M, Tokunaga E et al. Inducible nitric oxide synthase plays a role in LPS-induced hyperglycemia and insulin resistance. Am J Physiol Endocrinol Metab 2002; 282: E386-E394.

6 Stith RD, McCallum RE. Down regulation of hepatic glucocorticoid receptors after endotoxin treatment. Infect Immun 1983; 40: 613-621.

7 Oguri S, Motegi K, Iwakura Y, Endo Y. Primary role of interleukin-1 alpha and interleukin-1 beta in lipopolysaccharide-induced hypoglycemia in mice. Clin Diagn Lab Immunol 2002; 9: 1307-1312.

8 Christ B, Nath A. Impairment by interleukin 1 beta and tumour necrosis factor alpha of the glucagon-induced increase in phosphoenolpyruvate carboxykinase gene expression and gluconeogenesis in cultured rat hepatocytes. Biochem J 1996; 320(Pt 1): 161-166.

9 Maitra SR, Gestring ML, El-Maghrabi MR, Lang CH, Henry MC. Endotoxin-induced alterations in hepatic glucose-6-phosphatase activity and gene expression. Mol Cell Biochem 1999; 196: 79-83.

10 Altarejos JY, Montminy M. CREB and the CRTC co-activators: sensors for hormonal and metabolic signals. Nat Rev Mol Cell Biol 2011; 12: 141-151. 
11 Liu Y, Dentin R, Chen D et al. A fasting inducible switch modulates gluconeogenesis via activator/coactivator exchange. Nature 2008; 456: 269-273.

12 Dentin R, Liu Y, Koo SH et al. Insulin modulates gluconeogenesis by inhibition of the coactivator TORC2. Nature 2007; 449: 366-369.

13 Screaton RA, Conkright MD, Katoh Y et al. The CREB coactivator TORC2 functions as a calcium- and cAMP-sensitive coincidence detector. Cell 2004; 119: 61-74.

14 Mizock BA. Alterations in fuel metabolism in critical illness: hyperglycaemia. Best Pract Res Clin Endocrinol Metab 2001; 15: 533-551.

15 Ye H, Arron JR, Lamothe B et al. Distinct molecular mechanism for initiating TRAF6 signalling. Nature 2002; 418: 443-447.

16 Chen ZJ. Ubiquitin signalling in the NF-kappaB pathway. Nat Cell Biol 2005; 7: 758-765.

17 Rieser E, Cordier SM, Walczak H. Linear ubiquitination: a newly discovered regulator of cell signalling. Trends Biochem Sci 2013; 38: 94-102.

18 Chen Z, Hagler J, Palombella VJ et al. Signal-induced site-specific phosphorylation targets I kappa B alpha to the ubiquitin-proteasome pathway. Genes Dev 1995; 9: 1586-1597.

19 Wang Y, Li G, Goode J et al. Inositol-1,4,5-trisphosphate receptor regulates hepatic gluconeogenesis in fasting and diabetes. Nature 2012; 485: 128-132.

20 Aramburu J, Garcia-Cozar F, Raghavan A, Okamura H, Rao A, Hogan PG. Selective inhibition of NFAT activation by a peptide spanning the calcineurin targeting site of NFAT. Mol Cell 1998; 1: 627-637.

21 Aramburu J, Yaffe MB, Lopez-Rodriguez C, Cantley LC, Hogan PG, Rao A. Affinity-driven peptide selection of an NFAT inhibitor more selective than cyclosporin A. Science 1999; 285: 2129-2133.

22 del Rey A, Besedovsky H. Interleukin 1 affects glucose homeostasis. Am J Physiol 1987; 253: R794-R798.

23 Meinz H, Lacy DB, Ejiofor J, McGuinness OP. Alterations in hepatic gluconeogenic amino acid uptake and gluconeogenesis in the endotoxin treated conscious dog. Shock 1998; 9: 296-303.

24 Filkins JP, Cornell RP. Depression of hepatic gluconeogenesis and the hypoglycemia of endotoxin shock. Am J Physiol 1974; 227: 778-781.

25 Knowles RG, McCabe JP, Beevers SJ, Pogson CI. The characteristics and site of inhibition of gluconeogenesis in rat liver cells by bacterial endotoxin. Stimulation of phosphofructokinase-1. Biochem J 1987; 242: 721-728.

26 McCallum RE, Berry LJ. Effects of endotoxin on gluconeogenesis, glycogen synthesis, and liver glycogen synthase in mice. Infect Immun 1973; 7: 642-654.

$27 \mathrm{He} \mathrm{B}$, Cruz-Topete D, Oakley RH, Xiao X, Cidlowski JA. Human glucocorticoid receptor beta regulates gluconeogenesis and inflammation in mouse liver. Mol Cell Biol 2016; 36: 714-730.

28 Hill MR, Stith RD, McCallum RE. Interleukin 1: a regulatory role in glucocorticoid-regulated hepatic metabolism. J Immunol 1986; 137: 858-862.

29 Hill MJ, Suzuki S, Segars JH, Kino T. CRTC2 is a coactivator of GR and couples GR and CREB in the regulation of hepatic gluconeogenesis. Mol Endocrinol 2016; 30: 104-117.

30 Meszaros K, Bagby GJ, Lang CH, Spitzer JJ. Increased uptake and phosphorylation of 2-deoxyglucose by skeletal muscles in endotoxin-treated rats. Am J Physiol 1987; 253: E33-E39.

31 Meszaros K, Lang CH, Bagby GJ, Spitzer JJ. Contribution of different organs to increased glucose consumption after endotoxin administration. J Biol Chem 1987; 262: 10965-10970.

32 Meszaros K, Lang CH, Bagby GJ, Spitzer JJ. Tumor necrosis factor increases in vivo glucose utilization of macrophage-rich tissues. Biochem Biophys Res Commun 1987; 149: 1-6.

33 Qi L, Saberi M, Zmuda E et al. Adipocyte CREB promotes insulin resistance in obesity. Cell Metab 2009; 9: 277-286.

34 Dentin R, Hedrick S, Xie J, Yates J III, Montminy M. Hepatic glucose sensing via the CREB coactivator CRTC2. Science 2008; 319: 1402-1405.

35 Song Y, Altarejos J, Goodarzi MO et al. CRTC3 links catecholamine signalling to energy balance. Nature 2010; 468: 933-939.

36 Jansson D, Ng AC, Fu A, Depatie C, Al Azzabi M, Screaton RA. Glucose controls CREB activity in islet cells via regulated phosphorylation of TORC2. Proc Natl Acad Sci USA 2008; 105: 10161-10166.

37 Luan B, Goodarzi MO, Phillips NG et al. Leptin-mediated increases in catecholamine signaling reduce adipose tissue inflammation via activation of macrophage HDAC4. Cell Metab 2014; 19: 1058-1065.

38 Wang Y, Vera L, Fischer WH, Montminy M. The CREB coactivator CRTC2 links hepatic ER stress and fasting gluconeogenesis. Nature 2009; 460: 534-537.

(Supplementary Information is linked to the online version of the paper on the Cell Discovery website.)

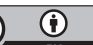

This work is licensed under a Creative Commons Attribution 4.0 International License. The images or other third party material in this article are included in the article's Creative Commons license, unless indicated otherwise in the credit line; if the material is not included under the Creative Commons license, users will need to obtain permission from the license holder to reproduce the material. To view a copy of this license, visit http://creativecommons.org/licenses/by/4.0/

(C) The Author(s) 2016 